\title{
The old nova CP Puppis: a carbon nova and asynchronous polar? ${ }^{\star}$
}

\author{
A. Bianchini ${ }^{1}$, T. Saygac ${ }^{2}$, M. Orio ${ }^{3}$, M. della Valle ${ }^{4}$, and R. Williams ${ }^{5}$ \\ ${ }^{1}$ Department of Astronomy, University of Padova, vicolo dell'Osservatorio 3, 35122 Padova, Italy \\ e-mail: antonio.bianchini@unipd.it \\ 2 Department of Astronomy and Space Sciences, Istanbul University, Istanbul \\ 34119, Turkey \\ 3 INAF - Osservatorio Astronomico di Padova, vicolo dell'Osservatorio 5, 35122 Padova, Italy \\ ${ }^{4}$ INAF - Osservatorio Astronomico di Napoli, Salita Moiariello 16, 80131 Napoli, Italy \\ 5 Space Telescope Science Institute, 3700 San Martin Drive, Baltimore, MD 21218, USA
}

Received 12 May 2011 / Accepted 21 January 2012

\section{ABSTRACT}

\begin{abstract}
Context. CP Pup (Nova Pup 1942) showed outburst and quiescent characteristics indicating a very massive white dwarf, yet the standard spectroscopic dynamical analysis assuming an accretion disk yields an extremely low value for the white dwarf mass. However, some physical parameters and the accretion geometry are still poorly known.

Aims. The nova was spectroscopically monitored between 1988 and 1996. We analyzed the whole data set in order to re-determine the spectroscopic period and examine its stability. We also looked for chemical anomalies in the spectrum.

Methods. We obtained the radial velocity curves for the hydrogen and helium lines from our last better quality 1996 run. The mean 1996 spectrum yields information on the chemical composition of the binary. We also searched the mean period using the multi-year data set.

Results. From the radial velocities of our complete data set we derive the most probable mean spectroscopic period and tentatively suggest revised ephemeris. However, we demonstrate that the period is intrinsically unstable. We show that a standard accretion disk model does not explain all the spectroscopic features observed nor the variability of the spectroscopic period. We suggest that only interpreting the system as a slightly asynchronous polar would fit the data. The mean optical spectrum of CP Pup shows also an enhanced carbon abundance. Non solar abundances in the accreted material are unexpected and interesting, confirming that the nature of the secondaries of old novae should be studied more in detail. In fact, in CP Pup, as in other novae, the enhanced carbon is an important clue to the pre-outburst evolution, implying that the secondary was heavily polluted with carbon and helium during the common envelope phase of the pre-cataclysmic binary by a relatively massive primary that filled its Roche lobe during the third dredge up on the asymptotic giant branch.
\end{abstract}

Key words. accretion, accretion disks - stars: magnetic field - novae, cataclysmic variables

\section{Introduction}

CP Pup exploded in 1942 as one of the brightest and fastest of galactic classical novae. It only bears similarities to V1500 Cyg, they were both fast evolving "disk novae" (Della Valle \& Livio 1998) with ejecta characterized by large expansion velocities and exceptionally large ranges in brightness. The post-nova luminosities never reached their pre-outburst quiescent values but remained in a sort of standstill. Shaefer \& Collazzi (2010) found that CP Pup might still be more than five magnitudes brighter than its pre-explosion level. The two old novae have virtually identical spectra and also show similar photometric behaviors. The major difference between them is the proven strong magnetic field of V1500 Cyg, which dominates its geometry. In fact, Stockman et al. (1988) found that a fraction of the light from V1500 Cyg shows circular polarization modulated with the orbital period, while Cropper (1986) and Scaltritti \& Orio (1999, at CASDEO, unpublished), did not detect such a feature in CP Pup. The nature of CP Pup is still debated - although the presence of

* CP Pup mean spectrum is available in electronic form at the CDS via anonymous ftp to cdsarc.u-strasbg.fr (130.79.128.5) or via

http: //cdsarc.u-strasbg.fr/viz-bin/qcat?]/A+A/539/A94 a magnetic white dwarf (WD) seems to be very likely (Balman et al. 1995; Orio et al. 2001).

\subsection{The spectroscopic period}

Bianchini et al. (1985a,b,c, hereafter BFS85a-c, respectively) found the spectroscopic period $P=0.06115 \mathrm{~d}$. CP Pup then appeared as the first nova with an orbital period below the 2-3 h period gap for cataclysmic variables (CV; Warner 1995). Duerbeck et al. (1987, hereafter DSD) found $P=0.0614215 \mathrm{~d}$, O'Donoghue et al. (1989, hereafter OWWG) reported either 0.06141 or $0.06148 \mathrm{~d}$, while Patterson \& Warner (1998) assumed a mean stable spectroscopic period $P=0.06143(3) \mathrm{d}$. Bianchini et al. (1990, hereafter BFS90), using additional spectroscopic data obtained in 1988 and 1989, measured a mean period of $0.061375 \mathrm{~d}$, but the folded radial velocity curves show large scatter in the data points. Finally, White et al. (1993, hereafter WHH) observed a night-to-night slightly variable period and derived a mean value of $0.06129 \mathrm{~d}$. Thus, spectroscopic observations have not yet converged on a reliable value of the true orbital period. An additional puzzle is the small mass of the WD given by all the dynamical solutions using the radial velocities, e.g. Barrera \& Vogt (1989) and OWWG. Quite on the contrary, the high outburst amplitude can only be explained by a massive WD. 


\subsection{The photometric period}

The photometric behavior of the old nova is also complex. In 1985, Warner (1985) discovered a photometric modulation with a period of either 0.06614 or $0.06196 \mathrm{~d}$, and suggested that since the orbital period is close to that of the SU UMa dwarf novae, the observed modulation might be a superhump phenomenon. This interpretation might be also supported by the fact that the protracted "standstill" state of the post-nova could very well be associated with enhanced mass transfer. OWWG observed $0.06138 \mathrm{~d}$ in 1986 and $0.06154 \mathrm{~d}$ in 1988. Even more discrepant photometric periods were found by Diaz \& Steiner (1991), who measured $0.0641 \mathrm{~d}$ but claimed they observed the 88 -min orbital period of a strongly magnetized WD, and by WHH who detected a much longer period of $0.06834 \mathrm{~d}$, i.e. $11 \%$ longer than their spectroscopic period. Patterson \& Warner (1998) measured a stable modulation at $0.06143 \mathrm{~d}$, consistent with the spectroscopic/orbital period, but also noted the presence of an additional transient signal at $P=0.0625 \mathrm{~d}$, which was instead unstable in both amplitude and frequency. The photometric period excess of $2 \%$ over the spectroscopic period is reminiscent of superoutburst regimes, while the instability of the signal does not argue in favor of an intermediate polar interpretation. An alternative hypothesis is that the unstable longer photometric period component is arising from the rotation of a magnetic white dwarf slightly out of spin-orbit synchronism (Warner 1985; Diaz \& Steiner 1991; Balman et al. 1995).

\subsection{The accretion disk}

OWWG found saddle-shaped line profiles with the peaks corresponding to separations of $550 \mathrm{~km} \mathrm{~s}^{-1}$, and that the variation of the radial velocities of the line peaks is in phase with that of line wings. They also found a decrease of the radial velocities semiamplitude, $K_{1}$, from line center to wings, consistently with BFS85c who reported that radial velocity variations were better shown by line peaks than by line barycenters. In addition, OWWG noted that the variations of the ratios between the violet and the red intensity peaks of the emission lines $(V / R)$ showed an enhanced blue peak of the lines at phase 0.25 , while the red peak appeared enhanced at phase 0.75 . This suggested the presence of a region of enhanced emission on the side of the disk directly opposite the usual location of the bright-spot. This picture was confirmed by the Doppler tomography performed by WHH that, in fact, placed the most intense line emission region on the side of the disk opposite that of the secondary star with respect to the WD primary. WHH however concluded that Doppler tomography failed to clearly distinguish between a DQ Herculis model or an SU Ursae Majoris model for the disk. An enhanced emitting region placed on the side of the accretion disk opposite the secondary was also observed by Shafter et al. (1986) in the dwarf nova SW UMa, probably a DQ Her intermediatepolar system, and by Gilliland et al. (1986) for the dwarf nova WZ Sge, which is known to be an SU UMa system. Thus, the dynamics and geometry of the CP Pup system are still unclear.

\subsection{The uncertain mass of the WD}

The outburst characteristics of CP Pup suggest a high mass WD (e.g. Prialnik \& Kovetz 1995). As shown by WHH, the published estimates of $K_{1}$ range from 70 to $146 \mathrm{~km} \mathrm{~s}^{-1}$, while the mass estimates of the WD $\leq 0.2 M_{\odot}$ are consistenly too low to support a nova outburst, according to current models. For this reason, several authors claimed that the observed $K_{1}$ does not correctly represent the motion of the white dwarf. Recently, new Mass - Radius correlations, obtained by assuming the critical mass ratio for the creation of superhumps, predict a smaller mass for the secondary of CP Pup and a larger mass for the primary. The semi-empirical calibrations derived by Patterson et al. (2005) and Knigge (2006) suggest a mass of the secondary $M_{2} \sim 0.082-0.083 M_{\odot}$, while Pearson (2006), from the period excess, predicts $q=0.085, M_{2}=0.070 M_{\odot}$ and a mass of the primary $M_{1}=0.830(7) M_{\odot}$, the latter value being more reasonable for the WD of a nova system. However, from an observational point of view the problem of the large observed value of $K_{1}$ is still debated and basically unsolved.

\subsection{The X-Ray behavior}

The quiescent X-ray luminosity of CP Pup $\sim 2 \times 10^{32} \mathrm{erg} \mathrm{s}^{-1}$ is higher than average quiescent novae (Orio et al. 2001) and more typical of Intermediate Polars. CP Pup was only the third nova for which an $X M M$-Newton grating observation could be obtained (Orio et al. 2001). Several prominent lines of high ionization stages of $\mathrm{O}, \mathrm{Ne}$ and Fe were detected, and the X-ray spectrum is well fitted as a stationary cooling flow of shocked accreted material continuously replenished by accretion, at a temperature possibly as high as $70 \mathrm{keV}$ (Orio et al. 2001). The maximum temperature is an indication of the accretion shock, and its high value is very interesting because it is indicative of the large gravitational potential of a high mass white dwarf. The $\mathrm{K}$ iron features and the $6.4 \mathrm{keV}$ fluorescent line are also detected. Orio et al. (2001) were able to derive the two most important parameters that determine the evolution of a white dwarf burning hydrogen in a shell (e.g. Fujimoto 1982; Yaron et al. 2005): a high white dwarf mass exceeding $1.1 M_{\odot}\left(1.3 M_{\odot}\right.$ if accretion is only through a disk, not funneled by a strong magnetic field) and a moderately low mass accretion rate $\left(\dot{m}<1.6 \times 10^{-10} M_{\odot} \mathrm{yr}^{-1}\right)$. These authors also confirmed Balman et al. (1995) result that the $\mathrm{X}$-ray flux is modulated with the spectroscopic period. They concluded that the X-ray spectra are explained by a high mass white dwarf accreting at low rate, in agreement with nova theory.

\subsection{New observations}

In an attempt to address some of the ambiguities surrounding CP Pup we present previously unpublished spectroscopic observations of the old nova and discuss them together with previous results obtained by BFS85a and BFS90. In Sect. 2 we describe our new observations and the data reduction. In Sect. 3 we present the revised ephemeris of the mean spectroscopic period and discuss the main spectrophotometric features observed with respect to theoretical models of CVs. Conclusions are summarized in Sect. 4.

\section{The observations and data reduction}

Twenty five spectra of the old nova CP Pup were taken on 14-15 March 1996, and seven spectra on 21-22 September 1996 with the $1.5 \mathrm{~m}$ ESO telescope and the $\mathrm{B} \& \mathrm{C}$ spectrograph. The spectral range is $\lambda \lambda 4200-5100 \AA$ and the spectral resolution is $2.5 \AA$. Exposure times ranged from 20 to $25 \mathrm{~min}$. Due to a more efficient CCD, the signal-to-noise ratios (S/N) of the $1996 \mathrm{spec}-$ tra are 25 to $35,2-3$ times better than the $\mathrm{S} / \mathrm{N}$ of the spectra obtained in our past runs with the same $1.5 \mathrm{~m}$ ESO telescope. Data reduction of the old nova and standard stars was performed 
A. Bianchini et al.: The old nova CP Pup

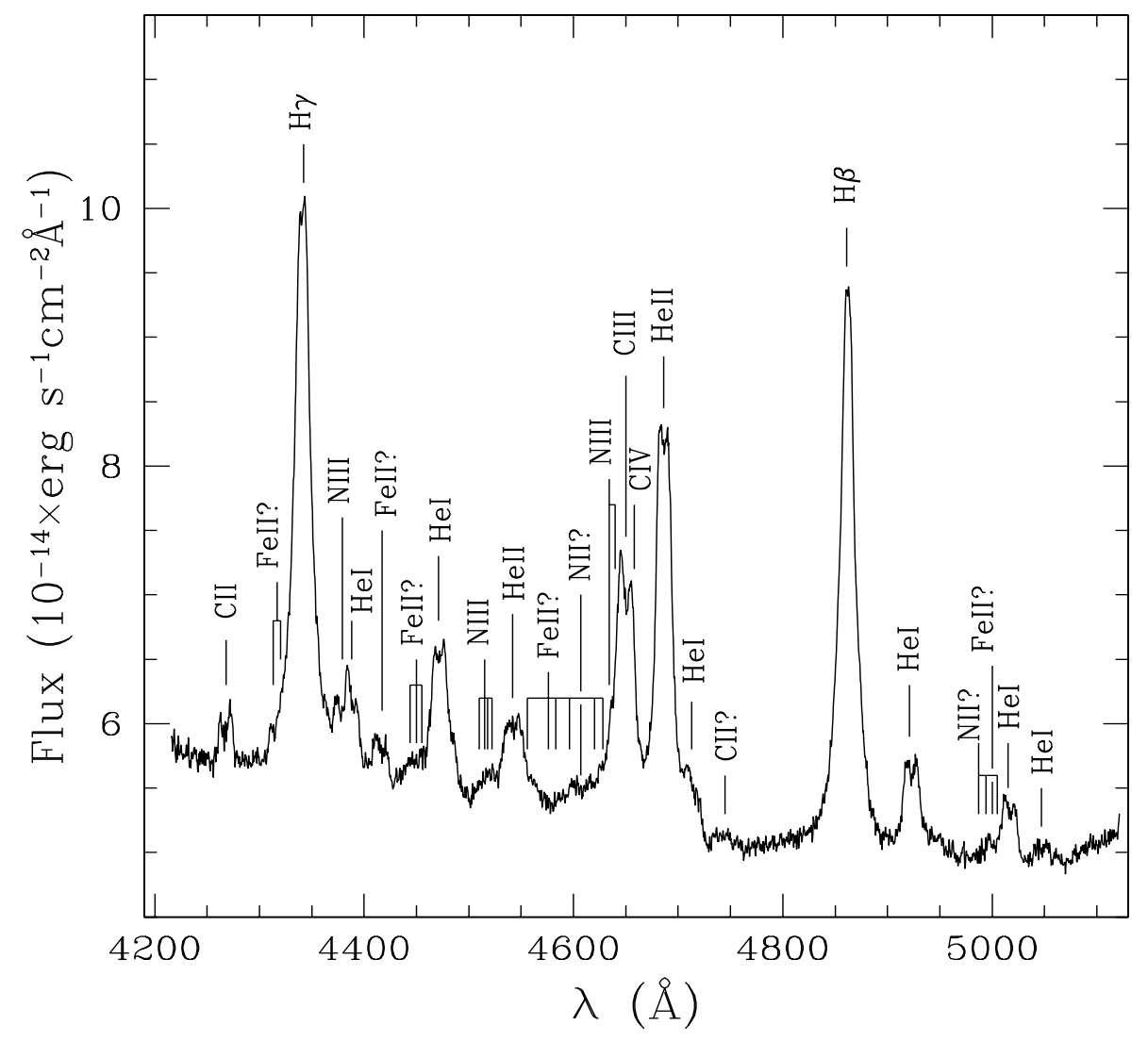

Fig. 1. Sum of 1996 spectra show a possible enhancement of carbon, similar to that deduced for the old nova V840 Oph (Schmidtobreick et al. 2003). Amongst other similarities, these two old novae show a strong C IV $\lambda 5804$ emission (see spectra published by Duerbeck et al. 1987; Williams \& Ferguson 1982). using the standard IRAF software package. The radial velocities and the equivalent widths of the emission lines were measured by fitting single and/or multiple Gaussians to the line profiles. Fourier analysis as well as the $\chi^{2}$ method were used to search for periodic variations.

\section{Results}

\subsection{The spectrum and line identification}

The mean spectrum of CP Pup is shown in Fig. 1. It represents the sum of all spectra obtained from the first run of our 1996 ESO observations because these spectra showed the best $\mathrm{S} / \mathrm{N}$. The $\mathrm{S} / \mathrm{N}$ of the mean continuum spectrum is $\sim 120$. The continuum shows a distribution law $F_{\lambda} \propto \lambda^{-\alpha_{\lambda}}$ with $\alpha_{\lambda} \sim 1.0$. A similar slope, in the same spectral range, is also shown by the spectrum published by Williams \& Ferguson (1983; upper panel of their Fig. 4) although at shorter wavelengths the emission Balmer jump mimics a steeper slope. Assuming an interstellar absorbtion $A_{\mathrm{V}}=0.3 \mathrm{mag}$ (Cohen \& Rosenthal 1983) and using the extinction law derived by Fitzpatrick (1999) we derive $\alpha_{\lambda} \sim 1.3$. This is a lower value than predicted by integrating the radiation of a black body disk (Lynden-Bell 1969) with $\alpha_{\lambda}=2.3$. We calculate that the mass transfer rate that would produce in CP Pup an optically thick accretion disk which is also thermally stable (i.e. $T_{\text {eff }}$ is everywhere higher than $7000 \mathrm{~K}$ so that no dwarf nova-like activity is possible) up to an outer radius $\sim 0.7 \times R_{\mathrm{RL} 1}$ is $1.6 \times 10^{-10} M_{\odot} \mathrm{yr}^{-1}, R_{\mathrm{RL} 1}$ being the mean radius of the primary Roche lobe (Warner 1995). But this is also the upper limit to the mass transfer rate derived from X-ray observations (Orio et al. 2001). Nevertheless, the post-nova is about one hundred times brighter then the pre-nova and has been also considered to be in a continuous superhump phase. In principle, this would strongly suggest a relatively high accretion rate capable of producing an extended optically thick accretion disk. However, the smaller slope of the continuum and the uncertain value of the actual mass transfer rate does not allow any precise conclusion. Thus, CP Pup may not be hosting a fully developed optically thick accretion disk.

The brightest emission lines observed in the mean spectrum of the old nova are those of hydrogen, helium and carbon ions. Some unresolved fainter emissions are tentatively identified as due to C II, N II, N III and Fe II. Table 1 reports the main ions identified and, when possible, the flux, equivalent width (EW) and full width at half maximum (FWHM) of the emission lines. The associated errors are on the order of $10 \%$.

The last column of Table 1 gives the velocity separation of the two peaks of line profiles showing double peaks. This velocity difference can be interpreted as twice the projected rotational velocity $V_{\text {out }} \sin i$ of the outer regions of the accretion disk, or of a ring, or as the infall velocities of two magnetically driven accretion streams.

\subsection{Line profiles and intensities}

Double peaked profiles that could be produced by accretion disks, rotating rings, or magnetic accretion columns are clearly shown by the emission lines of He I, He II, C II and C III. The mean separation between their peaks is consistent with that found by OWWG and would correspond to a rotational velocity of the outer regions of the disk of $270 \mathrm{~km} \mathrm{~s}^{-1}$. The profiles of $\mathrm{H} \beta$ and $\mathrm{H} \delta$ appear saddle-shaped only in some of our spectra, while their mean profiles in Fig. 1 look rather single peaked. This suggests that the hydrogen emission lines are produced in more than one distinctive region of the binary system and may also vary in time. The velocity inferred from the wings of the lines, that could be associated with the rotation of the inner 
Table 1. Widths and intensities of the main emission lines observed.

\begin{tabular}{lccccc}
\hline \hline Ion & $\begin{array}{c}\lambda \\
(\AA)\end{array}$ & $\begin{array}{c}\text { Flux } \\
\left(\mathrm{erg} \mathrm{s}^{-1} \AA^{-1} \mathrm{~cm}^{-2}\right)\end{array}$ & $\begin{array}{c}\text { EW } \\
(\AA)\end{array}$ & $\begin{array}{c}\text { FWHM } \\
(\AA)\end{array}$ & $\begin{array}{c}2 \times V \sin i \\
\left(\mathrm{~km} \mathrm{~s}^{-1}\right)^{a}\end{array}$ \\
\hline H $\gamma$ & 4340 & $8.5 \times 10^{-13}$ & 15.0 & 19.0 & - \\
$\mathrm{H} \beta$ & 4861 & $8.1 \times 10^{-13}$ & 16.0 & 18.8 & - \\
$\mathrm{He} \mathrm{I}$ & 4471 & $2.5 \times 10^{-13}$ & 4.5 & 22 & 508 \\
$\mathrm{He} \mathrm{I}$ & $4388 b$ & $1.0 \times 10^{-13}:$ & $1.8:$ & $17:$ & - \\
He I & $4713 b$ & $1.0 \times 10^{-13}:$ & $2.2:$ & $20:$ & - \\
He I & 4922 & $1.4 \times 10^{-13}$ & 2.5 & 17.2 & 476 \\
He I & $5016 b$ & $8.6 \times 10^{-14}$ & 1.8 & 17.3 & 505 \\
He I & 5048 & $2.5 \times 10^{-13}:$ & $0.5:$ & $19:$ & - \\
He II & 4542 & $1.5 \times 10^{-13}$ & 3.0 & 19 & 533 \\
He II & 4686 & $6.5 \times 10^{-13}$ & 12.3 & 18.5 & 448 \\
C II & 4268 & $6.0 \times 10^{-14}$ & 1.1 & 15.8 & 632 \\
C III & 4650 & $4.7 \times 10^{-13}:$ & $8.8:$ & $23.8:$ & 528 \\
C IV & $4660 b$ & - & - & - & - \\
N III & $4640 b$ & - & - & - & - \\
\hline
\end{tabular}

Notes. ${ }^{(a)}$ From double peaked profiles. ${ }^{(b)}$ Blended with other lines. ${ }^{(:)}$Uncertain value. ${ }^{(-)}$Not measured.

regions of an accretion disk or with the terminal velocity of magnetically controlled accretion flows, is not easy to measure due to the blending of the lines. However, the broad component of the $\mathrm{H} \beta$ profile indicates $\sim 2000 \mathrm{~km} \mathrm{~s}^{-1}$ while OWWG measured only $1350 \mathrm{~km} \mathrm{~s}^{-1}$.

The inverted Balmer decrement $\mathrm{H} \gamma / \mathrm{H} \beta \sim 1.0$ and the EW's of the two hydrogen lines fit in the grid of accretion disk models calculated by Williams (1991) assuming an optically thick disk with midplane hydrogen density $\log N \sim 13$ and temperature $\sim 15000 \mathrm{~K}$.

The detection of several low excitation lines indicates the presence of low temperature emitting regions. On the other hand, the existence of high temperature regions is confirmed by the emission of CIII and He II (see Table 1), where HeII/H $\beta=0.76$, and also by X-ray and UV observations (Orio et al. 2001). Actually, the existence in the old nova of more than one source of radiation with distinct temperatures was previously suggested from time-resolved multicolor photometry performed by Diaz \& Steiner (1991).

The expanding envelope of CP Pup, analyzed by Williams (1982), Escalante \& Dalgarno (1991), and Smits (1991a,b) is characterized by low temperatures and nitrogen abundances 400 to 700 times the solar abundance. The central object, instead, shows enhanced carbon emission features. We first notice that the $\lambda 4645$ blend shown in Fig. 1 is strongly dominated by the C III $\lambda 4650$ multiplet, that also appears double peaked. Actually, the N III $\lambda 4640$ emission is only barely visible on the blue wing of the stronger C III emission component. Williams \& Ferguson (1983) stressed that, in old nova remnants, the individual contribution by the N III $\lambda 4640$ and C III $\lambda 4650$ multiplets in forming the broad $\lambda 4645$ blend may greatly vary from object to object and that the N III $\lambda 4640$ emission is likely to be caused by the selective excitation of the Bowen process. The weakness of this Bowen fluorescence emission component in the spectra of CP Pup suggests that the required large optical depth and low density conditions, or the required velocity coincidence between distinct emitting regions, do not occur within this binary system. That CP Pup is a carbon-rich old nova is also confirmed by the presence in our spectrum of the emission of C II $\lambda 4267$ and by the C IV $\lambda 5804$ emission that can be seen in the spectra published by Williams \& Ferguson (1983) and DSD. The presence of evolved material in the nova remnant is also validated by the strength of emission lines of N V, O I, C III, Si IV, C IV, He II,
$\mathrm{N}$ IV and possibly $\mathrm{O} \mathrm{V}$, detected with the SWP camera of IUE (Orio et al. 2001). The carbon abundance however cannot be determined because the C IV line at $\lambda 5804$ is out of our spectral range.

\subsection{The radial velocity curve}

The radial velocities of the hydrogen and He II emissions were measured by multiple Gaussian fitting of their profiles. Hydrogen lines have been fitted with two Gaussians to measure the radial velocities of their narrow and broad components. The He II $\lambda 4686$ emission is partially blended with that of CIII $\lambda 4650$. Its double peaked profile is clearly seen in the mean spectrum of the nova but individual spectra show rather puzzling behavior and we could identify the two peaks only in a few cases. Similar behavior was noted with the hydrogen lines but we did not find any correlation between the two different features. For these reasons, radial velocities of the He II emission were measured taking the centers of the line profile at half height. Figure 2 plots the means of the radial velocities of the narrow and broad components of the hydrogen lines and the radial velocities of the centers of the HeII $\lambda 4686$ emission, folded with their common best period $P=0.061258 \pm 0.0001 \mathrm{~d}$. The period is the most probable amongst the aliases of the power spectrum produced by radial velocity variations and the sampling. Thus, the actual standard deviation is given by the width at half maximum of the power distribution of the aliases. Phases are given adopting the $T_{0}$ epoch that we will derive later.

The broad component of hydrogen lines shows a larger $K_{1} \sim$ $126 \pm 6 \mathrm{~km} \mathrm{~s}^{-1}$ than the $K_{1} \sim 73 \pm 4 \mathrm{~km} \mathrm{~s}^{-1}$ of the narrow component. OWWG found a decrease of $K_{1}$ from line center to wings, which appears to contradict our result. This discrepancy might be due to not having identified and measured the individual peaks of a line and also to the used different procedure to measure the radial velocities of the broad components. The He II $\lambda 4686$ emission shows $K_{1} \sim 55 \pm 5 \mathrm{~km} \mathrm{~s}^{-1}$, which is a smaller value than those derived from the hydrogen lines. This behavior seems to be confirmed by the results of OWWG. As a high excitation line, the He II emission is produced in the hotter regions of the accretion flow near the WD surface. For this reason it is assumed to better represent the orbital motion of the primary. Compared to the previously published values of $K_{1}$, all in the range $70-146 \mathrm{~km} \mathrm{~s}^{-1}$, the quite smaller $K_{1}$ of 


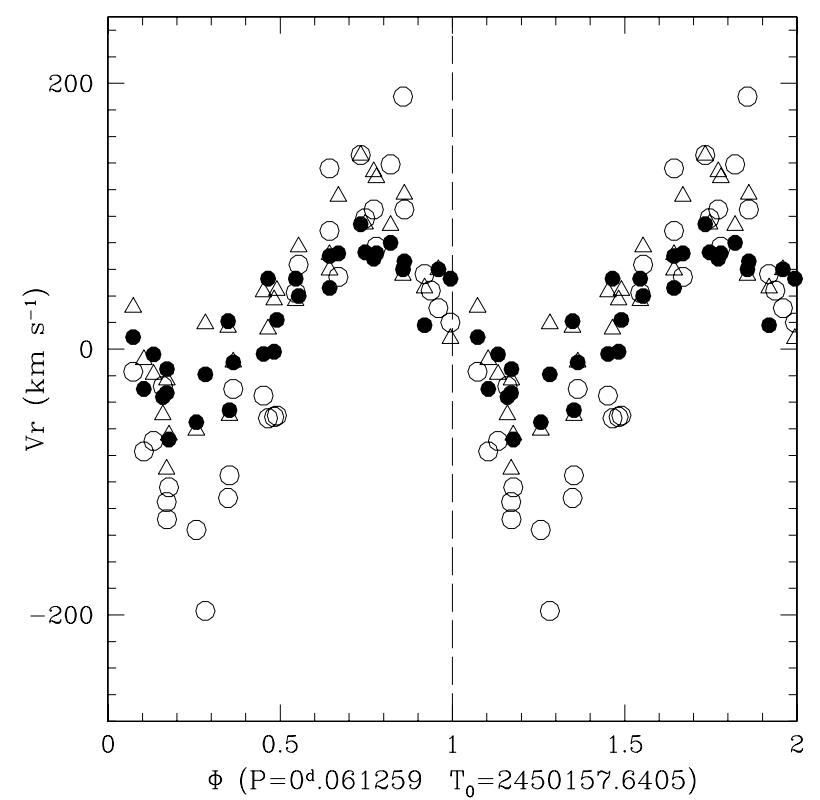

Fig. 2. Radial velocity curve derived from our 1996 ESO observations: open circles and triangles are the means of the velocities of $\mathrm{H} \beta$ and $\mathrm{H} \gamma$ emissions obtained using their broad and narrow components, respectively; filled circles are the radial velocities of the HeII $\lambda 4685$ emission measured at half line height (see text).

the He II emission found here is compatible with a dynamical mass of the WD $\sim 0.4 M_{\odot}$. This is a somewhat larger value than previous estimates, but still quite inadequate to account for a very fast nova like CP Pup. Hydrogen lines show a larger $K_{1}$, perhaps because they are emitted by more different portions of the accretion flow. The barycentric $\gamma$-velocities are $+2 \pm 6 \mathrm{~km} \mathrm{~s}^{-1}$ and $+35 \pm 4 \mathrm{~km} \mathrm{~s}^{-1}$ for the broad and the narrow components of hydrogen lines, respectively, and $+23 \pm 3 \mathrm{~km} \mathrm{~s}^{-1}$ for He II $\lambda 4686$.

OWWG noted that the $V / R$ variations suggest the presence of a region of enhanced emission that could be placed on a region directly opposite the usual location of the bright-spot in the accretion disk. A similar geometry was claimed by Shafter et al. (1986) for the dwarf nova SW UMa (suspected to be a DQ Her system) and by Gilliland et al. (1986) for the dwarf nova WZ Sge (known to be an SU UMa system). This picture for CP Pup was confirmed by Doppler tomography performed by WHH, although the actual structure of the reputed disk remains uncertain. However, the anomalous position of such a hot region could be also explained as the result of a magnetically driven accretion flow with ballistic trajectory that falls behind the WD as calculated by Schwarz et al. (2005) for the asynchronous polar BY Camelopardalis.

If we assume the presence of a brighter line emitting region in the binary system, like the hot spot of an accretion disk, that contributes to the observed radial velocities, then we may calculate both the radial velocity due to the orbital motion and that of the hot region. Some numerical tests to verify this approach will be discussed below. Within the superhump interpretation of the photometric period, the bright region might simply coincide with the elongated portion of an eccentric disk. In this case, due to the slow prograde precession of the tidally distorted disk the position of the intense line emission region should also slowly change, thus explaining the large range of the $K_{1}$ values reported in the literature as well as the night-to-night differences in the radial velocity parameters observed by WHH.

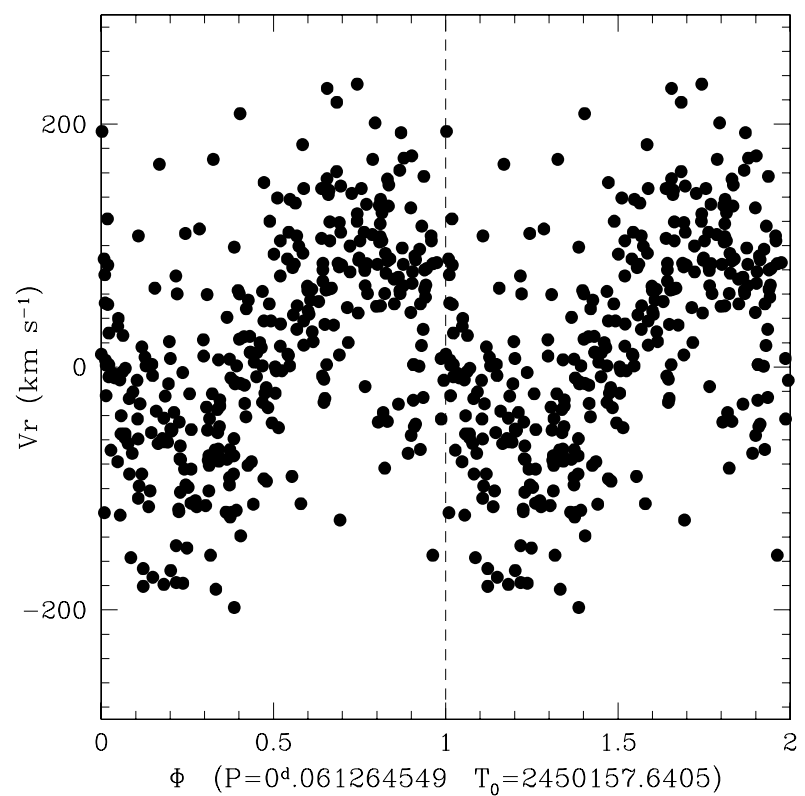

Fig. 3. Radial velocity curve obtained using our historical ESO set of data folded with the period of the main modulation detected.

We tried to derive a more precise mean spectroscopic period for CP Pup performing the Fourier analysis of our entire historical database. To further constrain the search, we added to our data base some fictitious radial velocities which ideally represent the plus to minus $\gamma$-crossing branch of the radial velocity curve according to the ephemeris given by WHH with $T_{0}=2446812.5961 \mathrm{HJD}$. The plot of all the radial velocities phased with the main period found $P=0.06126454(9 \pm 1) \mathrm{d}$ is shown in Fig. 3. We note that this mean period is reasonably close to that of Fig. 2. The large dispersion in the data points is not surprising because our previous spectra were obtained using CCDs with poor quantum efficiency in the blue spectral region, thus producing a very poor $\mathrm{S} / \mathrm{N} \sim 10$. Velocity dispersions of about $100 \mathrm{~km} \mathrm{~s}^{-1}$ can be also seen in the radial velocity curves published by DSD, while deviations of $100-200 \mathrm{~km} \mathrm{~s}^{-1}$ are shown by OWWG (their Fig. 4). A more physical interpretation of the velocity dispersion behavior is provided by Ferrario et al. (1993) for intermediate polars CVs in which the emission lines are assumed to originate in two accretion columns produced by the dipolar magnetic field of the WD.

In all cases, we tentatively present the following revised ephemeris

$$
T_{0}=2450157.640(5 \pm 1)+0.06126454(9 \pm 1) E(H J D) .
$$

The $K_{1}$ amplitude is $60 \pm 10 \mathrm{~km} \mathrm{~s}^{-1}$ and the $\gamma$-velocity is $+14 \pm$ $3 \mathrm{~km} \mathrm{~s}^{-1}$.

Figure 4 shows the power spectrum of our radial velocity data base implemented with some fictitious data points as explained above. Due to the complex sampling of the data we observe quite a forest of aliases. The main features shown in Fig. 4 are the frequencies that correspond to time intervals of 1 day and of 2-11 days, i.e. the lengths of individual runs. The narrow aliases are produced by time intervals of one or more years. We find that subtracting the highest frequency of the power spectrum, that corresponds to period $P_{1}=0.06126454 \mathrm{~d}$, we obtain residuals that still show a significant modulation with period $P_{2}=0.0615558 \mathrm{~d}$ and $K_{1}$ amplitude of about $41 \mathrm{~km} \mathrm{~s}^{-1}$. When we subtract both these periods the residuals basically show a flat noisy pattern (see Fig. 4). 


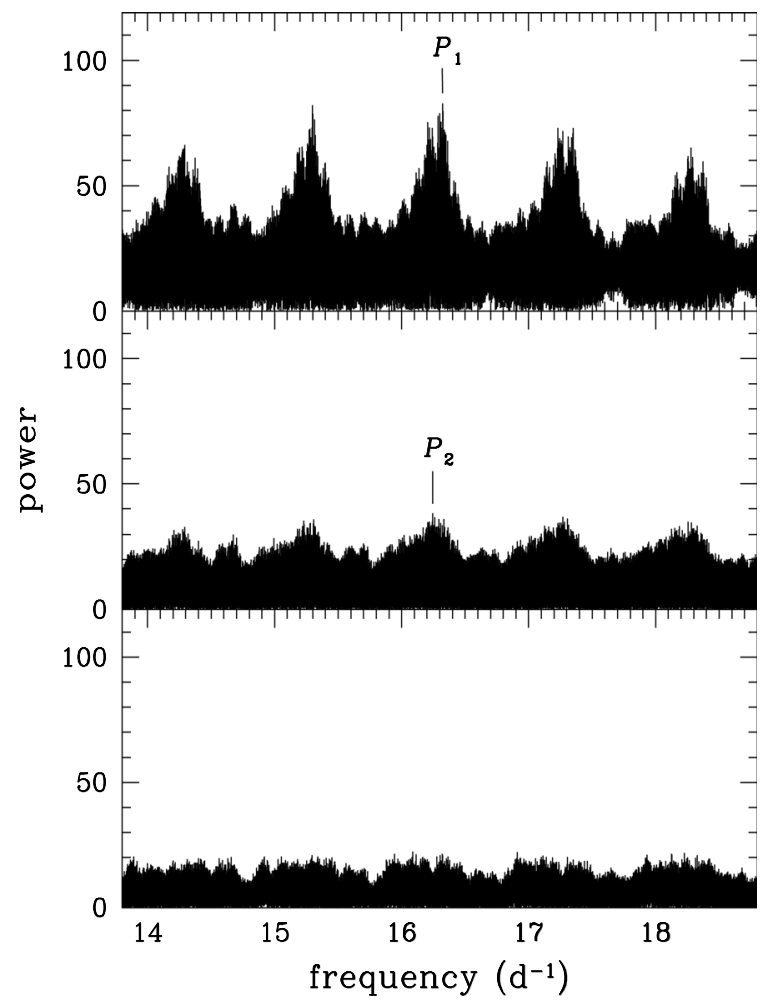

Fig. 4. A portion of the power spectrum of our radial velocities data base; top panel: the power spectrum of the original data shows a forest of aliases produced by the sampling, the highest of which corresponds to period $P_{1}=0.06126454 \mathrm{~d}$; middle panel: the power spectrum of the residuals after subtraction of $P_{1}$ still suggests a significant modulation with period $P_{2}=0.0615558 \mathrm{~d}$ (see text); bottom panel: when both periods are subtracted the residuals basically show a flat pattern.

To investigate the meaning of these two periods we must consider some details of the Fourier analysis procedure. It is well known that the frequencies produced by the sampling combine with the actual modulation of the data base producing a number of aliases in its power spectrum, the highest of which is usually assumed as the most probable period. In principle, when the main modulation is subtracted from the data all the other aliases should disappear. However, if the data time series is characterized by an intrinsically unstable period (like in the case of the so-called Quasi Periodic Oscillations), then the peaks of the power spectrum appear rather broad because there is not one monochromatic modulation that can simultaneously fit all the data. In this case, the subtraction of the main period does not leave totally flat residuals.

We notice that the secondary period $P_{2}$ is never detected in any of our individual annual observational runs because it would simply fall inside the broad profiles of the peaks of the power spectrum. Actually, it appears as a modest alias in the power spectrum of our whole data set but gets power only when the main period $P_{1}$ is subtracted from the data. All this means that, even using a long term data base, the Fourier analysis does not converge toward one single period. Actually, the power of the modulation is pathologically distributed over a range of frequencies or, we should better say, over a number of aliases. It is interesting to note that almost all published spectroscopic periods, including the periods we derive from our individual annual observational runs, cluster around period $0.0614 \mathrm{~d}$ that coincides with the mean of the two individual periods inferred by our whole data set. Only WHH observed a mean period of $0.06129 \mathrm{~d}$ that is close enough to our principal period and also reported a night-to-night variability from 0.06115 to $0.06148 \mathrm{~d}$. We finally note that our secondary period falls within the range of the photometric periods, almost coinciding with the photometric period of $0.06154 \mathrm{~d}$ reported by OWWG. The fact that the low frequency tail of the spectroscopic periods observed overlaps with the high frequency tail of the known photometric periods suggests that the two phenomena might not be physically separated.

We conclude that the spectroscopic period of CP Pup must be intrinsically variable. This behavior is more typical of asynchronous polars rather than of intermediate polars because when the orbital period and the spin period of the WD are only slightly different, the accretion flows must continuously detach and reconnect with the magnetic poles of the WD producing sudden changes in the observed radial velocities of the emission lines.

\subsection{Spectrophotometric variations}

Only modest variations are shown by the continuum and the line intensities on time scales of about $0.3 \mathrm{~d}$ while the EW's of He II and hydrogen lines suggest 5\% modulations around $0.064 \mathrm{~d}$, which are reminiscent of the longer period superhumps observed. The ratios $F(\lambda 4686) / F(\mathrm{H} \beta)$ present variations on time scales of 2.0 and $0.6 \mathrm{~d}$. We notice that periodicities of $0.5-2 \mathrm{~d}$ might be consistent with the precession periods of an eccentric disk or, better, of a ring as it could be inferred by the observed spectroscopic and superhump periods.

\subsection{The dynamical masses of the WD}

In principle, as long as the disk is not totally disrupted by the WD magnetic field, a close binary system can be described in the $M_{1^{-}}$ inclination plane by three curves that represent the constraints imposed to the dynamical solutions by the observed semiamplitude $K_{1}$ of the orbital motion of the primary and by the Keplerian velocities $V_{\text {in }}$ and $V_{\text {out }}$, derived from the emission line profiles of the material orbiting around the WD in the inner and the outer regions of the disk, respectively. We then assume i) the $M_{2}-P_{\text {orb }}$ relationship (Patterson 1984); ii) a maximum outer disk radius $\sim 0.8 \times R_{\mathrm{RL} 1}$; and iii) a dynamically stable inner disk radius $\sim 1.4 \times R_{\mathrm{WD}}$.

For an ideal CV system, a formal analytical solution would imply that these three curves intersect each other in one single point. However, the situation with CP Pup is far more complicated and partial solutions always indicate too low masses of the WD.

According to our latest observations, the $K_{1}$ of the HeII emission could be as low as $55 \mathrm{~km} \mathrm{~s}^{-1}$. In the $M_{\mathrm{WD}}$-inclination plane of Fig. 5 we plotted the line (dotted line) that satisfies the condition of the measured outer disk velocity $270 \mathrm{~km} \mathrm{~s}^{-1}$ and the line (short dashed line) constrained by the estimated inner disk velocity $V_{\text {in }}=2000 \mathrm{~km} \mathrm{~s}^{-1}$. A third solid curve shows that even the modest $K_{1}$ of the HeII lines is not sufficiently small to infer a mass for the WD that explains a very fast nova like CP Pup as well as the X-ray properties of the post-nova (Prialnik \& Kovetz 1995; Orio et al. 2001).

More satisfactory dynamical solutions are found assuming that the observed radial velocities are affected by the contribution of a hot spot. In fact, the two solid lines in the bottom of Fig. 5 are constrained by assuming a $10 \%$ contribution from a hot spot placed at $180^{\circ}$ with respect to the $W D$-secondary direction (see Doppler tomography by WHH), and a $20 \%$ contribution from a hot spot placed at $60^{\circ}$, that approximately corresponds 


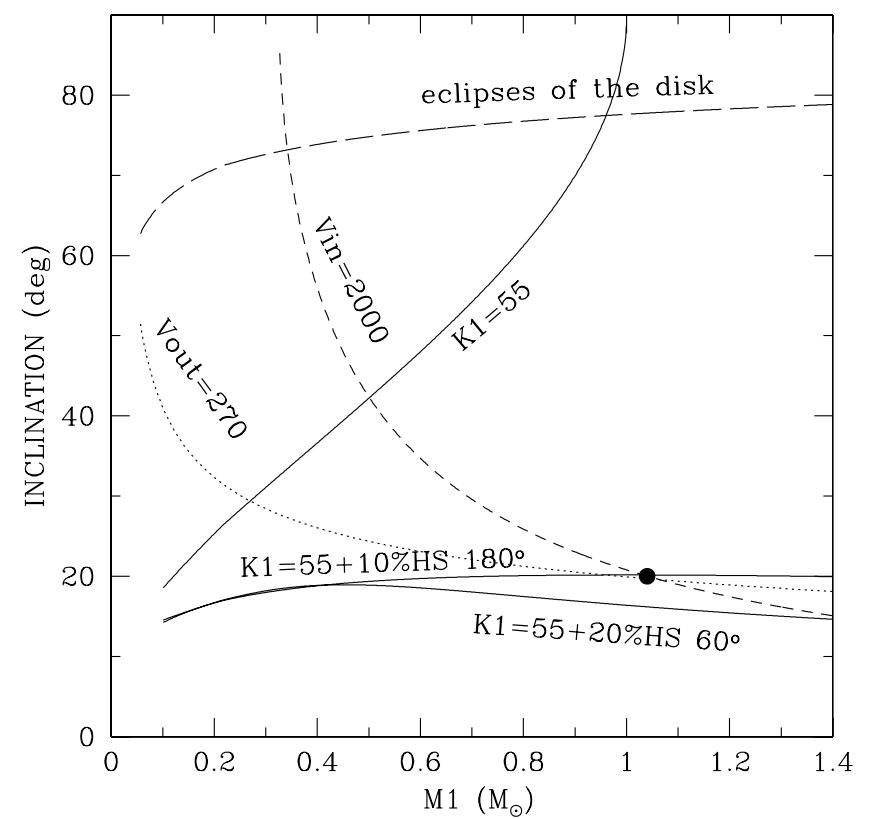

Fig. 5. The graph shows that even assuming the small $K_{1}$ derived from the HeII emission lines, a "standard" dynamical solutions of the spectroscopic parameters could lead to a reasonable mass for the WD only if we include the contribution from a hot spot (see text). The filled circle indicates a virtual analytical solution.

to the geometry of the standard model. In particular, the first curve would represent the analytical solution (filled circle in the figure) of a CP Pup system that contains a $1.05 M_{\odot}$ WD. The second curve might be suggestive of even more massive primaries. Thus, assuming the presence of an accretion disk, only solutions that incorporate the contribution from a hot spot can result in massive WD and a low inclination of the system that is compatible with the value of $\sim 30^{\circ}$ found by DSD and Szkody \& Feinswog (1988). It is however clear that by increasing the number of model parameters one can achieve a solution that fits most possible observational results. Obviously, another possibility would be a more drastic change of the whole accretion geometry. We then conclude that, most probably, the standard accretion disk model is not a fully realistic description of the accretion process in CP Pup.

\section{Discussion and conclusions}

From the analysis of our 1996 ESO spectroscopic observations of CP Pup, combined with the results obtained from our 1988 and 1989 previous runs, we measured a possible mean period of the radial velocities and tentatively derived new ephemeris. However, we found that the spectroscopic period is not stable. In fact, the presence of a second slightly longer period, detected only in the long term data base, can be only justified as the result of the complex sampling of the data and the the intrinsic variability of the spectroscopic period itself. We notice that the mean of the two periods found is $0.0614 \mathrm{~d}$ that coincides with most of the periods reported in the literature. This result seems to definitely confirm the unstable nature of the spectroscopic period.

We showed that if we assume the standard accretion disk geometry then the observed amplitude of the radial velocity curve, the separation of the two peaks of the emission lines and the widths of their wings, suggest very low masses for the WD contradicting the outburst behavior of the nova and its X-ray properties. We have demonstrated that even adopting the small
$K_{1} \sim 55 \mathrm{~km} \mathrm{~s}^{-1}$ radial velocity amplitude inferred by the He II emission lines, in order to obtain a more reasonable mass for the WD, $M_{\mathrm{WD}} \sim 1.05 M_{\odot}$ (or more), one has to introduce the contribution from a hot spot or, perhaps, assume a different accretion geometry, like magnetically driven accretion curtains or columns.

We noticed that the low frequency tail of the spectroscopic periods observed overlaps with the high frequency tail of the known photometric periods suggesting that the two phenomena are not totally physically separated. Warner (1985), Diaz \& Steiner (1991) and Balman et al. (1995) claimed that the unstable photometric period of CP Pup might arise from the rotation of a magnetic white dwarf slightly out of spin-orbit synchronism. Similarly, we suggest that the intrinsic instability of the spectroscopic period, together with the non-standard slope of the spectral continuum and the large dispersion seen in the radial velocity curve are better interpreted by an asynchronous polar nature of the old nova. In particular, since magnetic accretion curtains or funnels seen under an appropriate viewing geometry may give rise to emission line features that mimic those seen in a system with a full accretion disk (see Fig. 8 in Ferrario \& Wehrse 1999) we argue that lines formed under such conditions cannot be used to estimate the mass of the primary star. The asynchronous polar hypothesis might also explain the persistence of a hot region behind the WD that could be produced by the ballistic trajectory of a magnetically driven accretion flow (Schwarz et al. 2005).

In magnetic novae, an outburst can break down the spin/orbit synchronism. Schmidt et al. (1995) showed that the postoutburst spin period of the WD in the magnetic nova V1500 Cyg is about $2 \%$ shorter than the orbital one and that synchronization should be occurring in about $170 \mathrm{yr}$. The orbital modulation dominates the optical light curve that also shows a modulation at the $8 \mathrm{~d}$ beat period (Semeniuk et al. 1995). These authors suggested that accretion in V1500 Cyg occurs on both magnetic poles. A second asynchronous polar classical nova was found by Lipkin \& Leibowitz (2008) to be V4633 Sgr, i.e. nova Sagittarii 1998. These authors found that this nova followed a post-eruption evolution similar to that of V1500 Cyg. In both novae, immediately after the explosion the WDs showed a spin period slightly longer than the orbital period, probably due to a temporary increase in the primary's moment of inertia. Subsequently, the strong magnetic coupling and the contraction of the remnant envelope onto the WD surface led to a spin-up phase of the two primaries. The difference between V1500 Cyg and V4633 Sgr is that while the WD of Nova Cygni 1975 now has a spin period less than the orbital period, the WD of V4633 Sgr has a spin period which, for a number of reasons, remained longer than the orbital period. We suggest that the orbital and the spin periods of CP Pup are also slightly different from each other, although we are unable to decide which of the two cases above mentioned could be considered as more representative of the CP Pup system. As explained by Ferrario \& Wickramasinghe (1999), one should detect the beat phenomena between the orbital and the spin periods. However, since accretion onto the magnetic WD might be switching from one pole to the other because of the asynchronicity, as demonstrated by Silber et al. (1997), the beat periods cannot be simply determined from very long data sets but only from data that with sufficient time resolution can describe the spectrophotometric variations between two magnetic pole switches.

Finally, an important characteristic shown by the mean spectrum of CP Pup is its enhanced carbon content. Schmidtobreick et al. (2003), discussing the rediscovery of the old nova V840 Oph 1917, emphasized the existence of old novae with carbon 
dominated spectra and stressed their possible connection with magnetic systems. The presence of evolved gas in the accreted material argues for a more detailed investigation of the nature of the secondaries of CVs and especially of old novae. One possible interpretation is that the secondaries of carbon-rich novae were heavily polluted with carbon and helium during the common envelope phase of the pre-CV by relatively massive primaries that filled their Roche lobes during their third dredge up on the asymptotic giant branch (AGB). Ashes from the He-burning shell of the AGB primaries could be brought to the surface and ejected by thermal flashes. As a consequence, the convective secondaries could have stored large quantities of such evolved material that would be later on transferred back onto the WD primary. It is worthwhile to recall that Williams \& Ferguson (1982) have argued that also the presence of relatively strong He I emission is not fully understood unless one assumes that disks are overabundant in helium.

We propose higher resolution time-resolved spectroscopic observations of CP Pup to detect sudden changes in the radial velocities of the emission lines and to perform a better quantitative analysis of its elemental abundances. Simultaneous X-ray observations would also provide important information on the accretion physics and geometry.

Acknowledgements. We acknowledge Lilia Ferrario for suggestions and helpful discussion. T.S. wishes to thank the people of the Department of Astronomy of the University of Padova and of the Astrophysical Observatory of Asiago for their friendship and support. We thank two anonymous referees for their precious comments. We also thank the ESO staff at La Silla for their help during the observations. This work has been supported by the Italian MURST and by the UDP-15468 Project of Istanbul University.

\section{References}

Balman, S., Orio, M., \& Oegelman, H. 1995, ApJ, 449, L47

Barrera, L. H., \& Vogt, N. 1989, Rev. Mex. Astron. Astrofis., 19, 99

Bianchini, A., Friedjung, M., \& Sabbadin, F. 1985a, Inf. Bull. Variable Stars, 2650 (BSF85a)

Bianchini, A., Friedjung, M., \& Sabbadin, F. 1985b, in Proceedings of the Frascati Workshop, Multifrequency Behaviour of Galactic Accreting Sources, ed. F. Giovanelli (Frascati: CNR), 82 (BSF85b)

Bianchini, A., Friedjung, M., \& Sabbadin, F. 1985c, in Proceedings of ESA Workshop, Recent Results on Cataclysmic Variables, ed. W. R. Burke (Noordwijk: ESA SP-236), 77 (BSF85c)
Bianchini, A., Friedjung, M., \& Sabbadin, F. 1990, in Physics of Classical Novae, ed. A. Cassatella, \& R. Viotti (Berlin: Springer-Verlag), 61 (BSF90) Cohen, J. G., \& Rosenthal, A. J. 1983, ApJ, 268, 689

Cropper, M. 1986, MNRAS, 222, 225

Della Valle, M., \& Livio, M. 1998, ApJ, 506, 818

Diaz, M. P., \& Steiner, J. E. 1991, PASP, 103, 964

Duerbeck, H. W., Seitter, W. C., \& Duemmler, R. 1987, MNRAS, 229, 653 (DSD)

Escalante, V., \& Dalgarno, A. 1991, ApJ, 369, 213

Ferrario, L., \& Wehrse, R. 1999, MNRAS, 310, 189

Ferrario, L., \& Wickramasinghe, D. T. 1999, MNRAS, 309, 517

Ferrario, L., Wickramasinghe, D. T., \& King, A. R. 1993, MNRAS, 260, 149

Fitzpatrick, E. L. 1999, PASP, 111, 63

Fujimoto, M. Y. 1982, ApJ, 257, 767

Gilliland, R. L., Kemper, E., \& Suntzeff, N. 1986, ApJ, 301, 252

Knigge, K. 2006, MNRAS, 373, 484

Linden-Bell, D. 1969, Nature, 223, 690

Lipkin, Y. M., \& Leibowitz, E. M. 2008, MNRAS, 387, 289

O'Donoghue, D., Warner, B., Wargau, W., \& Grauer, A. D. 1989, MNRAS, 240, 41 (OWWG)

Orio, M., Covington, J., \& Oegelman, H. 2001, A\&A, 373, 542

Orio, M., Mukai, K., Bianchini, A., de Martino, D., \& Howell, S. 2009, ApJ, 690, 1753

Patterson, J. 1984, ApJS, 54, 443

Patterson, J., \& Warner, B. 1998, PASP, 110, 1026

Patterson, J., Kemp, J., Harvey, R., et al. 2005, PASP, 117, 1204

Pearson, K. J. 2006, MNRAS, 371, 235

Prialnik, D., \& Kovetz, A. 1995, ApJ, 445, 789

Schaefer, B. E., \& Collazzi, A. C. 2010, AJ, 139, 1831

Schmidt, G. D., Liebert J., \& Stockman, H. S. 1995, ApJ, 441, 414

Schmidtobreick, L., Tappert, C., Bianchini, A., et al. 2003, A\&A, 410, 943

Schwarz, R., Schwope, A. D., Staude, A., \& Remillard, R. 2005, A\&A, 444, 213

Semeniuk, I., Olech, A., \& Nalezẏty, M. 1995, A\&A, 45, 747

Shafter, A. W., Szkody, P., \& Thorstensen, J. R. 1986, ApJ, 308, 765

Smits, D. P. 1991a, MNRAS, 248, 193

Smits, D. P. 1991b, MNRAS, 248, 217

Szkody, P., \& Feinswog, L. 1988, ApJ, 334, 422

Silber, A. D., Szkody, P., Staude, A., et al. 1977, MNRAS, 290, 25

Stockman, H. S., Schmidt, G. D., \& Lamb, D. G. 1988, ApJ, 332, 282

Warner, B. 1985, MNRAS, 217, 1P

Warner, B. 1995, Cataclysmic Variable Stars (Cambridge: Cambridge Univ. Press)

White, J. C., Honeycutt, R. K., \& Horne, K. 1993, ApJ, 412, 278 (WHH)

Williams, R. E. 1982, ApJ, 261, 170

Williams, G. A. 1991, AJ, 101, 1929

Williams, R. E., \& Ferguson, D. H. 1982, ApJ, 257, 672

Williams, R. E., \& Ferguson, D. H. 1983, in Cataclysmic Variables and Related Objects, ed. Livio, \& Shaviv (Dordrecht: Reidel-Kluwer), IAU Coll., 72, 114 Yaron, O., Prialnik, D., Shara, M. M., \& Kovetz, A. 2005, ApJ, 623, 398 